\title{
Assessment of Renal Function from 3D Dynamic Contrast Enhanced MR Images using Independent Component Analysis
}

\author{
Frank G. Zöllner ${ }^{1,2}$, Marek Kocinski $^{3}$, Arvid Lundervold $^{2}$, Jarle Rørvik $^{1}$ \\ ${ }^{1}$ Department for Radiology, University of Bergen, 5021 Bergen, Norway, \\ ${ }^{2}$ Department of Biomedicine, University of Bergen, 5009 Bergen, Norway, \\ ${ }^{3}$ Institute of Electronics, Technical University of Lodz, 90-924 Lodz, Poland \\ Email: frank.zoellner@biomed.uib.no
}

\begin{abstract}
In this paper we present an automated, unsupervised, datadriven approach to assess renal function from 3D DCE-MR images. Applying independent component analysis to four different data sets acquired at different field strengths and with different measurement techniques, we show that functional regions in the human kidney can be recovered by a subset of independent components. Time intensity curves, reflecting perfusion in the kidney can be extracted from the processed data. The procedure may allow non-invasive, local assessment of renal function (e.g. glomerular filtration rate, GFR) from the image time series in future.
\end{abstract}

\section{Introduction}

Diagnosis of renal dysfunction is today based on blood test and urine sampling (e.g. plasma creatinine, creatinine clearance). These indirect measures are rather imperfect, since e.g. a significant change in creatinine level is only detectable until a $60 \%$ function loss has occurred. Furthermore, these measures do not give a split function between left and right kidney.

To overcome these limitations dynamic imaging of the kidneys is an emerging technique for a more accurate assessment of the local renal function [1, 2]. By using dynamic contrast enhanced magnetic resonance imaging (DCE-MRI), the passage of the contrast agent through the organ is reflected by intensity changes over time in the images. From the extracted regional time intensity curves, parameters such as blood flow and time of arrival of contrast agent can be estimated. Thus, a very precise assessment of renal function in the different compartments of the kidney is possible. However, this approach assumes proper motion correction (image registration) has been performed previous to the time course analysis, since the kidneys are non-rigid moving organs where complex voxel-displacements during data collection are caused by respiration and pulsations.

The emerging clinical interest in this kind of imaging studies stems partly from the fact that renovascular disease are rapidly increasing in the population, 
and can often progress to end stage renal disease (ESRD) requiring dialysis or kidney transplantation. Another diagnostic problem with relevance to the present study is early graft failure detection, where perfusion defects in the kidney transplant can occur at an early stage.

In this work we introduce an unsupervised, data-driven time course analysis method for regional assessment of renal function. By applying independent component analysis (ICA) to motion-corrected DCE MRI data we can automatically segment the 3D+time image into separate regions representing functional compartments of the kidney, e.g. the renal cortex, medulla, and pelvis. From each of these compartments we can obtain time intensity curves that provide valuable information about tissue perfusion and glomerular filtration, and thus renal function.

\section{State of the art and new contribution}

Assessment of renal function using DCE-MRI is typically based on manual or semi-automated delineation of regions of interest (ROIs) in the recorded images (e.g. [3]). This procedure is time consuming, expensive and error prone [4], and subject to intra- and inter-observer variations. Computational approaches can help overcome these limitations and provide more objective and reproducible results.

ICA of dynamic MR image acquisitions was first introduced by McKeown et al. [5], studying BOLD fMRI of brain. Since then, several different ICA algorithms and techniques have been proposed for the analysis of brain imaging data, including analysis of the 'default network' at resting state fMRI, and in group studies (cf. [6, 7]). To our knowledge, this is the first report using ICA in the study of human kidney DCE-MRI recordings obtained at 1.5 and 3T scanners with different pulse sequences and temporal and spatial resolutions (cf. [8]). The motivation for using ICA on images from moving kidneys was twofold (i) manual segmentation of functional compartments of the kidneys are time consuming and difficult since information is distributed in both time and space, (ii) renal physiology is relatively simple (compared to the brain), where we expect at least three sources contributing to the observed time courses, i.e. an early contrast enhancing cortical compartment, a late enhancing pelvic compartment, and an intermediate medullary compartment.

\section{Methods}

Briefly, ICA is a method for separating a multivariate signal $\mathbf{x}=\left(x_{1}, \ldots, x_{p}\right)$, into statistically independent components (or sources) $\mathbf{s}=\left(s_{1}, \ldots, s_{q}\right), q \leq p$. This can be formulated as $\mathbf{x}=\mathbf{A s}$ where $\mathbf{A}$ is the mixing matrix, and $\mathbf{A}$ and $\mathbf{s}$ are unknown. By inverting to $\hat{\mathbf{s}}=\mathbf{W} \mathbf{x}$, we can identify $\mathbf{W}$ as the unmixing matrix we wish to estimate, such that the statistical independence of the estimated sources $\hat{\mathbf{s}}$ is maximised, i.e. estimating maximum nongaussianity [9]. 
Table 1. Study data: $A=1.5 \mathrm{~T}$ Siemens Symphony, $\mathrm{B}=3.0 \mathrm{~T}$ Signa Excite GE

\begin{tabular}{cccccc}
\hline ID & Scanner & Sequence & Resolution $[\mathrm{mm}]$ & Size & Timing \\
\hline 1 & $\mathrm{~A}$ & FLASH & $1.48 \times 1.48 \times 3.00$ & $256 \times 256 \times 20 \times 20$ & not equidistant \\
2 & $\mathrm{~A}$ & VIBE & $1.48 \times 1.48 \times 3.00$ & $256 \times 256 \times 20 \times 118$ & 1 volume per $2.5 \mathrm{~s}$ \\
3 & $\mathrm{~B}$ & LAVA & $0.86 \times 0.86 \times 1.20$ & $512 \times 512 \times 44 \times 60$ & 1 volume per $3.0 \mathrm{~s}$ \\
4 & $\mathrm{~B}$ & LAVA & $1.72 \times 1.71 \times 2.40$ & $256 \times 256 \times 22 \times 60$ & 1 volume per $3.7 \mathrm{~s}$ \\
\hline
\end{tabular}

In our case we consider time courses $\mathbf{x}^{i}$ of the DCE-MRI data set for each voxel $i$ in a subregion containing the kidneys, where $p$ is the number of time frames. The task solved by the ICA algorithm is estimation of $\mathbf{s}^{i}$ (consisting of $q$ components) that might contribute to the functional relevant subregions of the kidney, or represent the surrounding tissue. Initially, the $4 \mathrm{D}$ data is preprocessed. First step is motion correction, applying a non-rigid registration procedure [10]. Thereafter, to discriminate between left and right kidney function, we used a kmeans clustering procedure, as in [11], to automatically separate the two kidneys.

For ICA analysis we used the fastICA algorithm to our preprocessed data. Prior to the calculation we reduced the dimension of the time series using principle component analysis (PCA) and whitening as suggested in [9]. A suitable number of PCs and ICs was eroded by applying the ICASSO method [12] which provides two measures on the stability of the ICA estimates (using clustering). The $\mathrm{I}_{q}$ coefficient measures the compactness of the clusters, the R-index $\left(\mathrm{I}_{R}\right)$ the similarity between the found ICs.

Furthermore, we projected the transformed data back into image space for assessing the location and spatial extension of the ICs, and for calculation of time intensity curves to identify functional parts of the kidney and verify the results qualitatively.

\section{Results}

We tested our approach on four data sets acquired from healthy volunteers. We used different field strength, pulse sequences, and spatial and temporal resolutions (cf. Tab. 1). A Gadolinium based contrast agent (Omniscan, GE Healthcare) were used in all experiments. The experiments were approved by the regional ethical committee.

For data analysis, the ICASSO and fastICA were applied to the 3D voxel time courses. By exploratory analysis of possible dimension reductions $\mathrm{I}_{R}$ showed a local minimum at 5 indicating a possible good solution [12]. In addition $\mathrm{I}_{q}$ showed high compactness values $(\geq 0.8)$ for all five independent components. As nonlinearity $g(u)$ used in the fixed point algorithm we select $g(u)=u^{3}$.

The resulting ICA signal was projected back into image space to visually inspect regions represented by the independent components. Figure 1 depicts the computed functional regions of the left kidney of data set 3 . For the renal cortex, medulla, and pelvis region the corresponding time intensity curves are plotted. The other two independent components (data omitted due to limited 
Fig. 1. Anatomically and functionally distinct regions and their corresponding time intensity curves resulting from ICA analysis of the left kidney of data set 3 . The renal compartments are superimposed on the original slice images from frame 10. The corresponding mean time intensity curves (bold) and the standard deviations (error bars) are plotted to the right. Top to bottom: cortex, medulla, and pelvis.
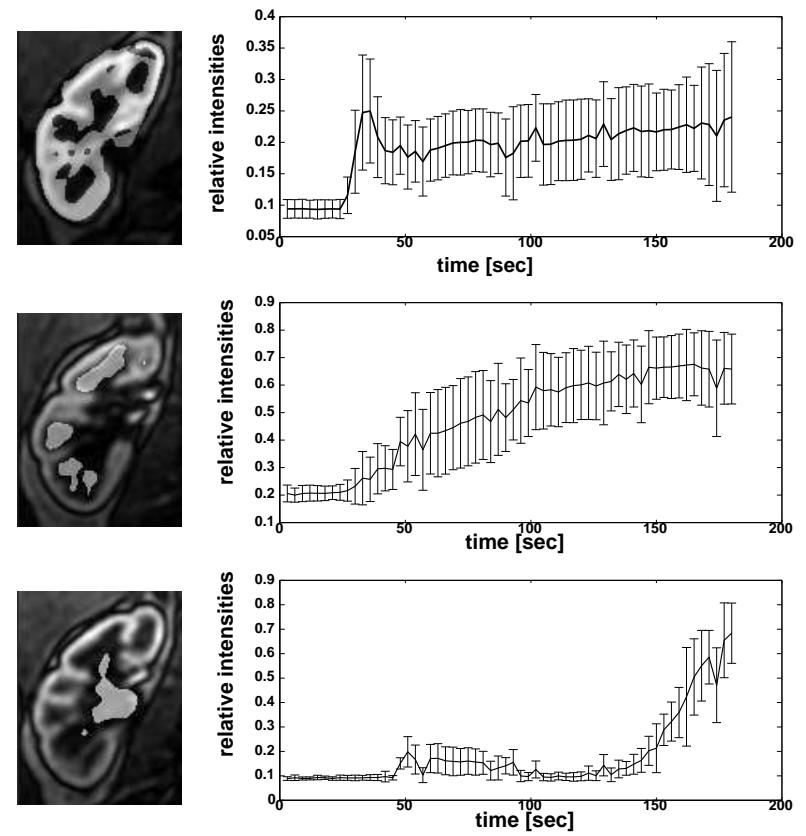

space) depict background voxels and voxels of partial volume effects. Similar results were obtained for the other data sets being analysed.

\section{Discussion}

We have presented an automated, unsupervised, data-driven ICA approach to assess renal function from 3D DCE-MR images of the human kidneys. Our results demonstrate that ICA is a powerful tool for segmenting time course information and functional 3D regions in the kidney. Using the ICASSO explorative visualisation method with bootstrapping, we found high statistical reliability and stability of the estimated independent components. Moreover, the regional mean time intensity curves in our healthy volunteers was close to those reported in [3]. A prerequisite for ICA analysis is proper volume-by-volume image registration since uncorrected large voxel displacements will introduce erroneous time courses and unreliable results [8]. However, the independent component analysis was also effective in detection of gross movements (e.g. when the breath-holding period ended), enabling assessment of registration performance, as the time courses belonging to these ICs had distinct spikes at times where misregistration occurred. 
Thus, it seems that periodic or stochastic voxel displacements is a source of information that can be captured by independent components much in the same way as background voxels or voxels subject to partial volume effects can be. Whether this information will be detected or not, will also depend on the number of independent components that are specified or being estimated.

Ongoing research is directed toward comparison of the independent component regions obtained by ICA analysis, and the segmentation results obtained by conventional k-means clustering of the time courses [11]. Using data-driven volume segmentation of the DCE-MR image recordings and transformation of timeintensity curves into time-concentration curves, we will explore local glomerular filtration rate (GFR) quantification using pharmacokinetic modelling (e.g. [13]).

\section{References}

1. Michaely H, Herrmann K, Nael K, et al. Functional renal imaging: Nonvascular renal disease. Abdominal Imaging 2006.

2. Prasad PV. Functional MRI of the kidney: tools for translational studies of pathophysiology of renal disease. Am J Physiol Renal Physiol 2006;290(5):F958-F974.

3. Huang AJ, Lee VS, Rusinek H. Functional renal MR imaging. Magn Reson Imaging Clin N Am 2004;12(3):469-86, vi.

4. de Priester JA, den Boer JA, Giele EL, et al. MR renography: an algorithm for calculation and correction of cortical volume averaging in medullary renographs. J Magn Reson Imaging 2000;12(3):453-459.

5. McKeown MJ, Makeig S, Brown GB, et al. Analysis of fMRI data by blind separation into independent spatial components. Human Brain Mapping 1998;6:160-188.

6. Calhoun VD, Adali T, Hansen LK, et al. ICA of Functional MRI Data: An Overview. In: Proc. 4th International Symposium on Independent Component Analysis and Blind Signal Separation (ICA2003); 2003. 281-287.

7. Calhoun VD, Adali T. Unmixing fMRI with independent component analysis. IEEE Eng Med Biol Mag 2006;25(2):79-90.

8. Zöllner FG, Kocinski M, Lundervold A. Assessment of kidney function from motion-corrected DCE-MRI voxel time-courses using independent component analysis. In: Int. Workshop on Mining Brain Dynamics. Bergen, Norway; 2006.

9. Hyvärinen A, Karhunen J, Oja E. Independent Component Analysis. Wiley Interscience; 2001.

10. Sance R, Rogelj P, Ledesma-Carbayo MJ, et al. Motion correction in dynamic DCE-MRI studies for the evaluation of the renal function. MAGMA 2006;19(Supplement 7):106-107.

11. Zöllner FG, Sance R, Anderlik A, et al. Towards quantification of kidney function by clustering volumetric MRI perfusion time series. MAGMA 2006;19(Supplement 7):103-104.

12. Himberg J, Hyvärinen A. Icasso: software for investigating the reliability of ICA estimates by clustering and visualization. In: Proc. 2003 IEEE Workshop on Neural Networks for Signal Processing (NNSP2003); 2003. 259-268.

13. Michoux N, Vallee JP, Pechere-Bertschi A, et al. Analysis of contrast-enhanced MR images to assess renal function. Magn Reson Mater Phy 2006;19:167-179. 